\title{
General paper \\ The Evolution of the Goss Texture in Silicon Steel
}

\author{
Dong Nyung LEE \\ School of Materials Science and Engineering, Seoul National University \\ San 56-1 Shinrim-dong, Kwanak-ku, Seoul 151-742, Korea
}

\begin{abstract}
The Goss orientation, $\{110\}<001>$, in about 3\% silicon steel has been the subject of speculation from its scientific and technological points of view. The grain oriented silicon steel is made by hot rolling at about $1300^{\circ} \mathrm{C}$, cold rolling, followed by annealing. The Goss texture is formed near the surface layer of a steel sheet when it is rolled in the $\alpha$ phase region at elevated temperatures, because the friction between the sheet and rolls tends to increase with increasing temperature, which in turn increases the shear deformation. The Goss texture remains unchanged even after recrystallization of steel. The Goss texture, which is not stable with respect to plane strain deformation, rotates toward the $\{111\}<112>$ orientation. The relaxed constraints (RC) model, in which shear strains parallel to the rolling direction may occur, simulates the formation of the $\{111\}<112>$ orientation. The $\{111\}<112>$ rolling component is known to lead to the Goss orientation after annealing. The strain energy release maximization model for recrystallization texture advanced by the author predicts the recrystallization texture of $\{441\}<118>$ from the $\{111\}<112>$ rolling texture. The $\{441\}<118>$ orientation yields the Goss orientation when the $\{441\}<118>$ orientation is expressed by the Gauss type scattering calculated with a half width angle of $12^{\circ}$. The model also predicts the fact that the Goss texture formed during rolling remains unchanged even after annealing.
\end{abstract}

Key words: Si steel, Recrystallization texture, Goss texture, Strain energy release maximization model

\section{INTRODUCTION}

The Goss orientation, $\{110\}<001>$, in about $3 \%$ silicon steel has been the subject of speculation from the scientific and technological points of view. The grain oriented silicon steel is made by hot rolling at about $1300^{\circ} \mathrm{C}$, cold rolling, followed by annealing. The Goss texture is formed near the surface layer of steel sheets when they are rolled in the $\alpha$ phase region at elevated temperatures. At elevated temperatures the friction between the sheet and rolls tends to increase with increasing temperature, which in turn increases the shear deformation and gives rise to the Goss texture. The Goss orientation is known to remain unchanged even after recrystallization of steel [1].

The Goss texture, which is not stable with respect to plane strain deformation, rotates toward the $\{111\}<112>$ orientation [2]. The Relaxed Constraints (RC) Taylor model, in which shear strains parallel to the rolling direction may occur, causes the formation of the $\{111\}<112>$ orientation [3]. The $\{111\}<112>$ rolling component is known to lead to the Goss orientation after recrystallization [2]. Raabe and Lücke [3] found that the orientation intensity of the Goss component in the recrystallization texture increased with increasing orientation intensity of the $\{111\}<112>$ component in the rolling texture. The above results can explain the "structure memory" mentioned in an article by Inokuti et al. [4]. Inokuti et al. found that the nucleation of $\{110\}<001>$ secondary grains takes place in the vicinity of the steel surface zone. The nuclei for secondary grains were found to be the large primary recrystallized grains of near $\{110\}<001>$ orientation which was formed by coalescence of several subgrains. They called the nucleation of the Goss orientation "structure memory" because the nucleation site was thought to be the site of the original Goss orientation formed during hot rolling.
Matsuo [5] attributed this phenomenon to the nucletion of the Goss orientation in shear bands which mainly occur in $\{111\}<112>$ host grains. Since the shear bands are inclined about $35^{\circ}$ about the axis along the transverse direction, the shear banding exactly rotates the $\{111\}<112>$ orientation into the Goss orientation. Raabe and Lücke claimed that the Goss nuclei as parts of shear bands could grow well into the surrounding deformed $\{111\}<112>$ grain during annealing since their $35^{\circ}<110>$ orientation relationship was the most favorable relationship for the growth of recrystallized nuclei [3]. However, their explanation is not convincing.

The author advanced a model for the evolution of recrystallization texture [6]. In the model, the absolute maximum internal stress direction in a deformed material becomes parallel to the direction of the minimum elastic

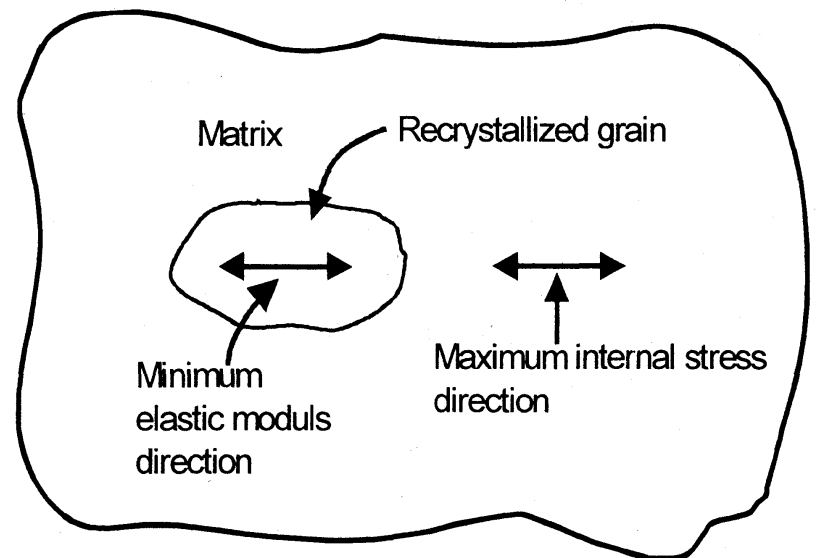

Fig. 1. Matrix and recrystallized grains constitute constant volume system, in which strain energy release can be maximized when absolute maximum internal stress direction is parallel to minimum elastic modulus direction of recrystallized grain. 


\section{Dong Nyung LEE}

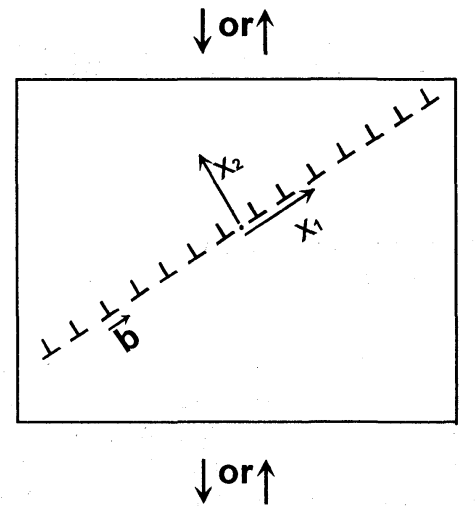

Fig. 2. Coordinates in edge dislocation array.

modulus of recrystallized grains, whereby the energy release during recrystallization can be maximized. This model is briefly described in the next section. This strain energy release maximization model has explained many recrystallization textures of fcc and bcc metals [6] such as the recrystallization textures of interstitial free (IF) steels [7], a plane strain compressed aluminum single crystal [8], shear rolled copper [9], aluminum [10], interstitial free steel sheets [1], plane strain compressed aluminum bicrystals with $\mathrm{S}$ orientation [11], the recrystallization textures of copper $[12,13]$ and silver [14] electrodeposits.

The purpose of this work is to discuss the thermal stability of the Goss orientation developed during rolling and the texture transformation from $\{111\}<112>$ to $\{110\}<001>$ based on the strain energy release maximization theory.

\section{STRAIN ENERGY RELEASE MAXIMIZATION MODEL}

In the model, the direction of absolute maximum inter-nal stress due to dislocations generated during deforma-tion becomes parallel to the minimum Young's modulus direction in recrystallized grains, whereby the strain en-ergy release during recrystallization can be maximized (Fig. 1). The concept is briefly explained in the following.

Supposed that a single crystal with a single slip system deforms under plane strain condition, then the dislocation array in the deformed crystal may be depicted as shown in Fig. 2. The stress fields of the array of an infinite num-ber of edge dislocations can be calculated by superposing the stress fields of isolated dislocations. Following Sutton and Ballufi [15], the stress fields of this array are given by

$$
\begin{gathered}
\sigma_{12}=-\sigma_{0} \sin X_{1}\left(\cosh X_{2}-\cos X_{1}-X_{2} \sin X_{2}\right), \\
\sigma_{11}=\sigma_{0}\left[2 \sinh X_{2}\left(\cosh X_{2}-\cos X_{1}\right)-X_{2}\left(\cosh X_{2} \cos X_{1}-1\right)\right],
\end{gathered}
$$

$$
\sigma_{22}=-\sigma_{0} X_{2}\left(\cosh X_{2} \cos X_{1}-1\right)
$$

where $X_{1}=2 \pi x_{1} / D, X_{2}=2 \pi x_{2} / D$, and $\sigma_{0}=-G b /[2 D(1-$ v) $\left.\left(\cosh X_{2}-\cos X_{1}\right)^{2}\right]$ with $G, b, v$, and $D$ being the shear modulus, the Burgers vector, Poisson's ratio and the dislocation spacing, respectively. As $x_{2} \rightarrow \pm \infty$, it is seen that $\sigma_{22}$ and $\sigma_{12}$ tend to vanish exponentially, but

$$
\sigma_{11} \rightarrow \frac{G b}{D(1-v)} \operatorname{sgn}\left(x_{2}\right)
$$

where $\operatorname{sgn}\left(x_{2}\right)=-1$ if $x_{2}>0$ and $\operatorname{sgn}\left(x_{2}\right)=1$ if $x_{2}<0$. The absolute maximum principal stress, $\left|\sigma_{\max }\right|=\mid\left(\sigma_{11}+\sigma_{22}\right) / 2$ $+\left[\left(\sigma_{11}-\sigma_{22}\right)^{2} / 4+\sigma_{12}^{2}\right]^{1 / 2} \mid$, approaches $\left|\sigma_{11}\right|$ exponentially as $\left|x_{2}\right|$ increases above $D / 2 \pi$. Therefore, the absolute maximum stress direction is parallel to the Burgers vector or the slip direction. A few examples of the principal stress distributions around parallel edge dislocations are shown in Fig. 3.

For multiple slip, the shear strains on active slip systems may not be equal. The dislocation density $\rho$ on a slip system can be approximated to increase with increasing shear strain $\gamma$ on it [16]. It follows that

$$
\left|\sigma_{11}\right| \approx\left|\sigma_{\max }\right| \propto 1 / D \propto \rho \propto \gamma .
$$

Therefore, the contribution of each active slip system to the absolute maximum stress direction will be proportional to the shear strain on it. When a crystal rotates during deformation, the shear strain rate $\mathrm{d} \gamma / \mathrm{d} \varepsilon$ on a slip system can vary with strain of specimen. In this case the contribution of each slip system to the absolute maximum stress will be proportional to total shear strain $\gamma$.

$$
\gamma=\int|\mathrm{d} \gamma / \mathrm{d} \varepsilon| \mathrm{d} \varepsilon
$$

If a deformation texture is stable, the ratio between the strains on the slip systems are independent of deformation and the contribution of the slip systems to the absolute maximum stress will be proportional to the shear strain rates on them. It is noted that the absolute maximum stress is an internal stress. They can be obtained from the deformation texture or from a model, which can simulate the deformation texture.

If a small volume of a uniaxially stressed body with fixed ends is replaced by the same volume of unstressed body as shown in Fig. 4, the strain energy of the system including the substituted region will be reduced. The released strain energy is represented as the area OAB in the figure. The released energy depends on Young's modulus of the substituted body. The released energy will be maximized, when Young's modulus of the substituted body is the minimum.

Since recrystallization is a displacement controlled process and the absolute maximum internal stress due to the dislocation array is approximated by the uniaxial stress, the deformed or fabricated matrix and the recrytallized grain are approximately equivalent to the stressed body and the substituted body, respectively, as shown in Fig. 4. In this case, we can state that the strain energy release can be maximized when the direction of absolute 


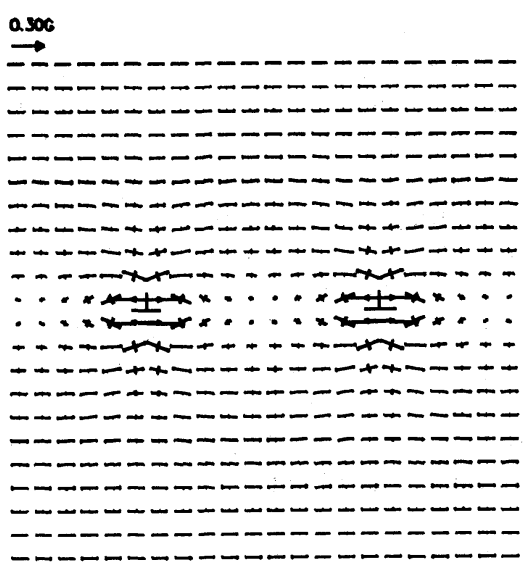

(a)

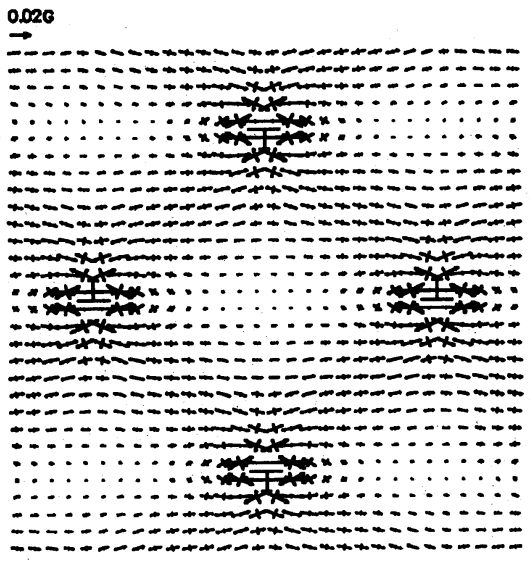

(b)

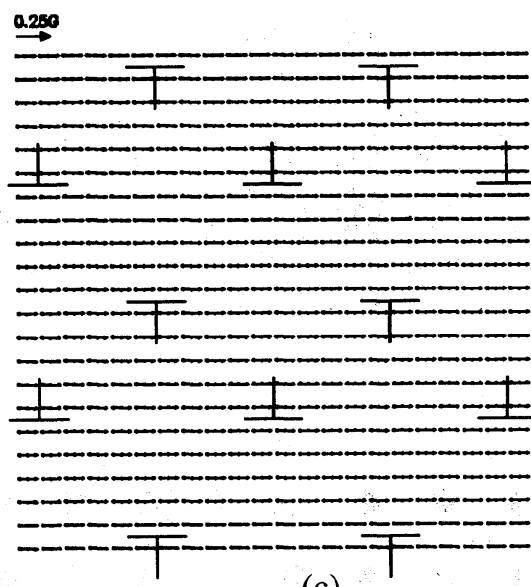

(c)

Fig. 3. Principal stress distributions around parallel edge dislocations calculated based on (a) 100 linearly arrayed dislocations with a dislocation spacing of $10 \mathrm{~b}$, (b) low energy array of $20 \times 20$ dislocations with a horizontal dislocation spacing of $20 b$, (c) low energy array of $100 \times 100$ dislocations with a horizontal dislocation spacing of $10 b . G$ is shear modulus and $b$ is Burger's vector.

(a)

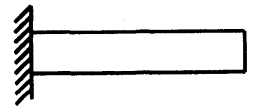

(b)

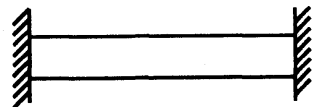

(c)

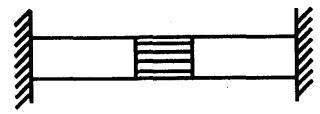

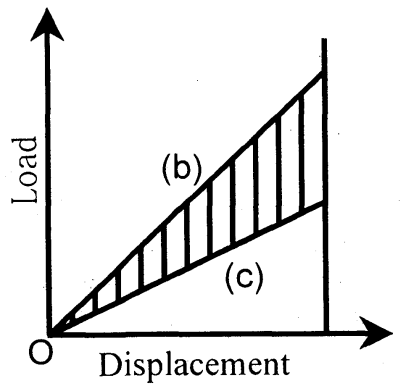

(d)

Fig. 4. When unstressed body (a) is elongated and its ends are fixed, strain energy of strained body (b) is represented by area OAC (d). When small portion of body (b) is replaced by unstressed material (c), strain energy of system is reduced to area $\mathrm{OBC}$ and released strain energy by area OAB.

maximum internal stress due to dislocations in the deformed or fabricated material becomes parallel to the minimum Young's modulus direction in recrystallized grains. Even though the real stress field is triaxial, this simple concept can be a starting point. To obtain the recrystallization texture in general, another orientation relation between the matrix and recrystallized grain is needed, which will be discussed in the following sections.

\section{THERMAL STABILITY OF GOSS ORIENTATION FORMED IN SURFACE LAYER}

The question is whether the Goss orientation could be stable during hot rolling. During hot rolling, recrystallization can take place, thereby the Goss orientation may change to a different orientation. Recently, Lee and Lee

[1] obtained an IF steel specimen with only the shear texture by a multi-layer warm rolling and discussed the evolution of its recrystallization texture. The material used was hot rolled $3.2 \mathrm{~mm}$ thick titanium-bearing extra low carbon IF steel sheet containing $0.002 \% \mathrm{C}, 0.045 \% \mathrm{Ti}$, $0.12 \% \mathrm{Mn}, 0.01 \% \mathrm{P}, 0.008 \% \mathrm{~S}$ and $0.034 \%$ sol.Al. The hot-rolled sheet was cold-rolled to $1.1 \mathrm{~mm}$ in thickness by several passes. Four of the $1.1 \mathrm{~mm}$ thick sheet were stacked, heated at $700^{\circ} \mathrm{C}$ for 30 minutes and rolled by $70 \%$ in the ferrite region without lubrication. The rolled specimen was quenched into $25^{\circ} \mathrm{C}$ water. Each layer was separated from the warm rolled sheet. In order to obtain a uniform shear texture, the surface layer was thinned from the inner surface to a half thickness by chemical polishing. The thinned surface and center layers were annealed at $750^{\circ} \mathrm{C}$ for $1 \mathrm{~h}$ in $\mathrm{Ar}$ atmosphere. Their recrystallization was examined by optical microscopy and hardness test. Texture measurement was carried out by the Schultz reflection method with $\mathrm{Zr}$ filtered Mo $\mathrm{K} \alpha$ radiation. ODFs (orientation distribution functions) were calculated by Bunge's series expansion method $\left(l_{\max }=22\right)$ using three incomplete pole figures, $\{110\},\{200\}$, and $\{211\}$ [17].

Figure 5 shows the measured (110) pole figures and ODFs of the outer and inner surfaces of the $75 \%$ warm rolled surface layer. The similarity of the two measured textures indicates that the texture of the layer is very uniform, and is approximated by the Goss texture of $\{110\}<001>$ and minor $\{112\}<111>$.

The deformation textures have been predicted by the following procedure [18]. The deformation gradient increments obtained from finite element analysis of rolling are used to obtain the strain increments. From the strain increments, a variation of texture through the thickness is calculated using the Taylor-Bishop-Hill model $[19,20]$. The $\{110\}<111>$ slip systems are assumed and rotation 

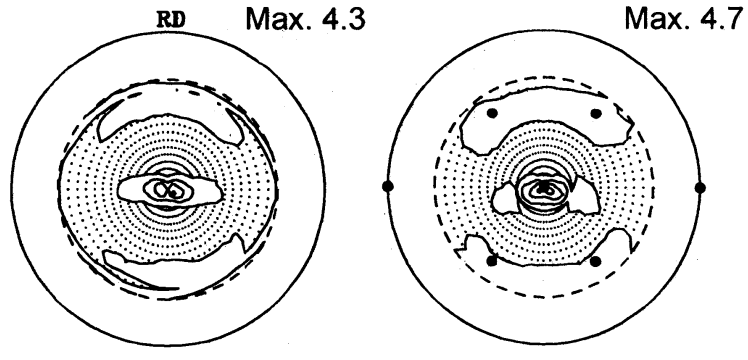

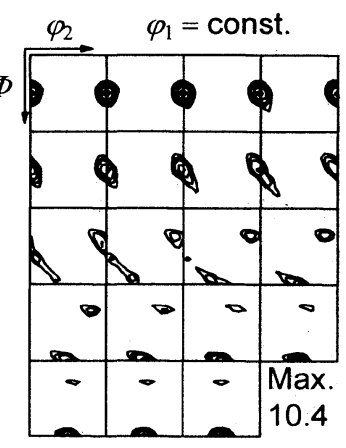

(a)

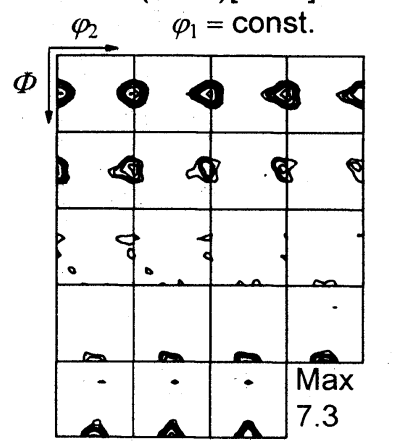

(b)
Fig. 5. Measured (110) pole figures and ODFs of (a) outer and (b) inner surfaces of surface layer.

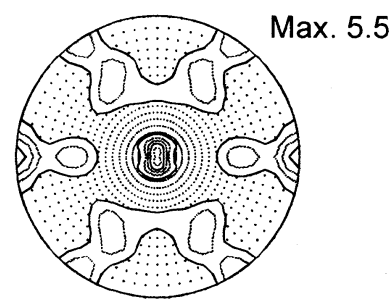

(a)

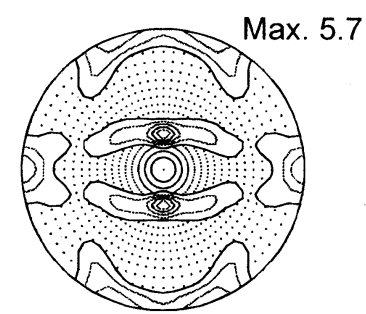

(b)
Fig. 6. Predicted (110) pole figures of (a) the surface and (b) the center layers of IF steel.

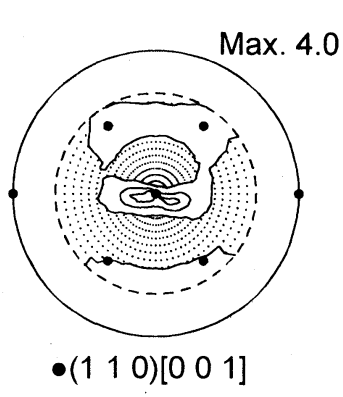

(a)

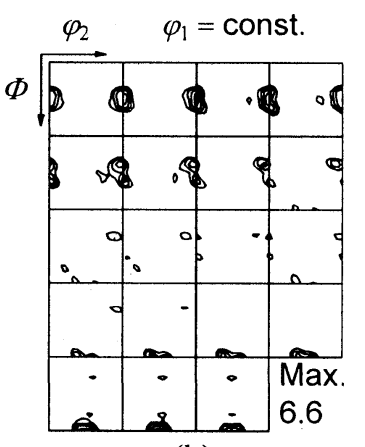

(b)
Fig. 7. (110) pole figure and ODF of the surface layer annealed after $75 \%$ warm rolling.

ambiguity is removed by the Renouard-Wintenberger model [21]. In bcc metals, slip occurs in the $\langle 111\rangle$ directions on the $\{111\},\{112\}$ and $\{123\}$ planes. But the slip plane is influenced by deformation temperature and the $\{110\}$ slip is favored between $T_{\mathrm{m}} / 4$ and $T_{\mathrm{m}} / 2$ [22]. Figure 6 shows the calculated (110) pole figures in the surface and center layers. The calculated textures are comparable with the measured textures.

Figure 7 show the texture of the chemically thinned surface layer when annealed at $750^{\circ} \mathrm{C}$ for $1 \mathrm{hr}$. The annealing texture of the surface layer is almost the same as the deformation texture in Fig. 5. Microstructures and hardness tests indicated recrystallization occurring after annealing [1]. The unchanged texture in the surface layer after annealing could be explained based on the strain energy release maximization model [1].

Table 1. The shear strain rate on each slip system as a function of $\varepsilon_{13} / \varepsilon_{11}$.

\begin{tabular}{ccccc}
\hline$\varepsilon_{13} / \varepsilon_{11}$ & $\mathrm{~d} \gamma_{1} / \mathrm{d} \varepsilon_{11}$ & $\mathrm{~d} \gamma_{2} / \mathrm{d} \varepsilon_{11}$ & $\mathrm{~d} \gamma_{3} / \mathrm{d} \varepsilon_{11}$ & $\mathrm{~d} \gamma_{4} / \mathrm{d} \varepsilon_{11}$ \\
\hline 0.5 & 1.225 & 1.225 & 0.245 & 0.245 \\
1.0 & 1.225 & 1.225 & 1.120 & 1.120 \\
1.2 & 1.225 & 1.225 & 1.466 & 1.466 \\
$\sqrt{ } 2$ & 1.225 & 1.225 & 1.837 & 1.837 \\
1.5 & 1.225 & 1.225 & 1.986 & 1.986 \\
\hline
\end{tabular}

In order to apply this model, the absolute maximum internal stress direction should be determined. The direction is obtained from the active slip systems during the deformation of the material. If the texture of the deformed material is stable, the active slip systems can be obtained from ideal major components of the texture and the deformation mode. The (110)[001] orientation was shown to be stable at $\varepsilon_{13} / \varepsilon_{11}=\sqrt{2}$ with subscripts 1 and 3 indicating the rolling and normal directions. Active slip systems for the (110)[001] crystal were calculated by the Taylor-Bishop-Hill theory, to be (1) $(0-1)[111]$, (2) (101)[111], (3) (110)[111], and (4) (100)[1 1 1]. For the multiple slip systems as in this case, the vector sum of slip directions is necessary. For the vector sum, the shear strain on each slip system calculated as a function of $\varepsilon_{13} / \varepsilon_{11}$ is given in Table 1 . The shear strain rates on slip systems (1) and (2) do not change, but those on slip systems (3) and (4) increase with increasing $\varepsilon_{13} / \varepsilon_{11}$. The slip systems having the higher shear strain rate give dominant contribution to the absolute maximum stress direction. As shown in Table 1, the slip systems (3) and (4) will give dominant contribution to the maximum stress direction for $\varepsilon_{13} / \varepsilon_{11}$ being equal to $\sqrt{2}$, which is condition for $\{110\}<001>$ to be stable. Therefore, the absolute maximum stress direction is a vector sum of [ 111$]$ and [1 111$]$, that is, [001]. The [001] direction is expected to be the absolute maximum internal stress direction. The [001] direction in deformed matrix will be parallel to one of the $\langle 100\rangle$ directions in recrystallized grains according to the strain energy release maximization model, because the minimum elastic modulus directions of bcc iron are the $\langle 100\rangle$ directions. Let the minimum elastic modulus direction in recrystallized grains be 
the [001]. Once the [001] direction is determined, another direction in the recrystallized grains may be obtained by taking symmetry and minimal atom shuffle during recrystallization into account. When the [001] direction is on a $\{110\}$ plane, the (110) plane in the present case, the symmetry condition is satisfied and atoms need to undergo minimum shuffle during recrystallization, because recrystallized grains and the deformed matrix will share the same (110) plane. As a result, the recrystallization texture will be (110)[001], which is also the deformation texture. It is interesting to note that one of the $<001>$ directions in the recrystallized grains, which is parallel to the absolute maximum normal stress direction in the deformed matrix, lies on one of the $\{110\}$ planes in the recrystallized grains. Thus, the main component of the shear texture, the Goss orientation, remains unchanged even after recrystallization. Therefore, the Goss texture formed during hot rolling of silicon steel is expected to be stable.

\section{EVOLUTION OF (111)[11 $]$ FROM GOSS ORIEN- TATION}

The Goss orientation is unstable with respect to plane strain deformation and rotated to the (111)[11 2 ] [3]. This texture change can be simulated by the relaxed constraints Taylor model.

\section{GOSS RECRYSTALLIZATION TEXTURE FROM (111) [112] DEFORMATION TEXTURE}

The evolution of the Goss orientation from the $\{111\}<112>$ orientation component has been explained by the strain energy release maximization model [23]. Slip systems of (110)[ $\overline{11} \overline{1}],(101)[11 \overline{1}]$ and (011)[11 1$]$ are calculated by the Relaxed Constraints Taylor model to be equally active in the (111)[11 $\overline{2}$ ] crystal undergoing the plane strain deformation. It is noted that the three slip directions are at acute angles with the extension direction of the crystal [111 $\overline{2}$ ]. The (101)[11 $\overline{1}]$ and (011)[11 $\overline{1}]$ slip

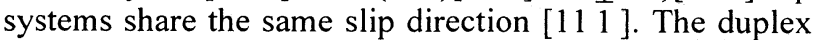
slip, with equal shear strains of $\gamma$ on each, is equivalent to a single shear strain of $2 \gamma \sin [0.5$ times angle between (101) and $(011)]=\gamma$ on the $(112)[11 \overline{1}]$ system [24]. Therefore the maximum internal stress direction is $[\overline{1} 1 \overline{1}]+[1 \overline{1} \overline{1}]+[11 \overline{1}]=[11 \overline{3}]$. According to the strain energy release maximization theory, this maximum stress

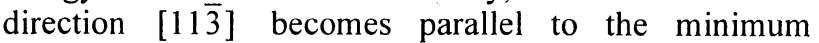
Young's modulus direction, $\mathrm{a}<100>$ direction in bcc iron, after recrystallization. Another direction must be obtained to determine the recrystallization texture. The direction may be obtained using conditions of symmetry and minimum shuffling of atoms during recrystallization. Let a $<100>$ direction be the [001] direction, then the [001] direction must be on the (100), (010) or (110) plane, taking the symmetry condition into account. One of them can be chosen by the minimum shuffling condition as already discussed in Chapter 3 .
The transverse direction of the (111)[11ㄹ] crystal is the $\left[\begin{array}{ll}1 & 1\end{array}\right]$ direction, along which there is no strain due to plane strain conditions. If the [332] direction, which is

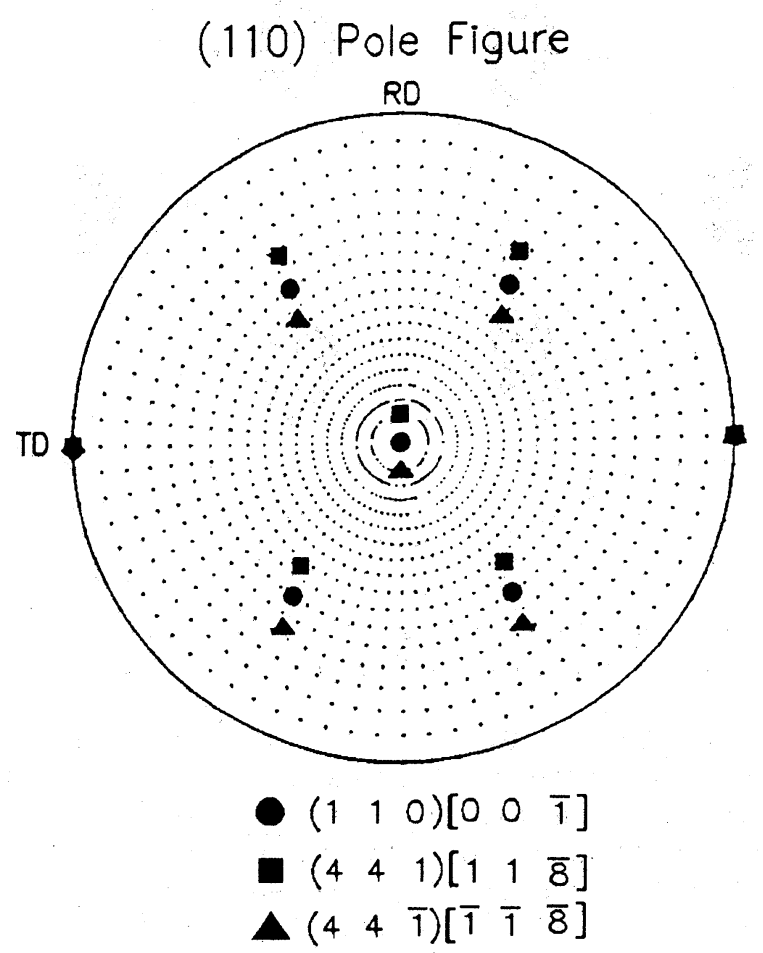

Fig. 8. $\{110\}<001>$ and $\{441\}<118>$ orientations in (110) pole figure

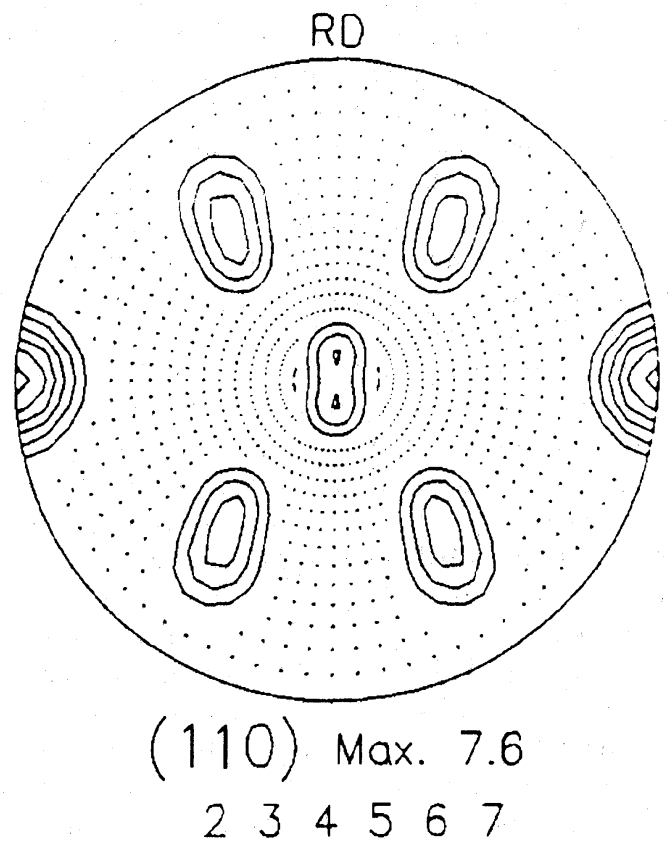

Fig. 9. (110) pole figure as Gaussian function of $\{441\}<118>$ orientation with a half width of $12^{\circ}$. 


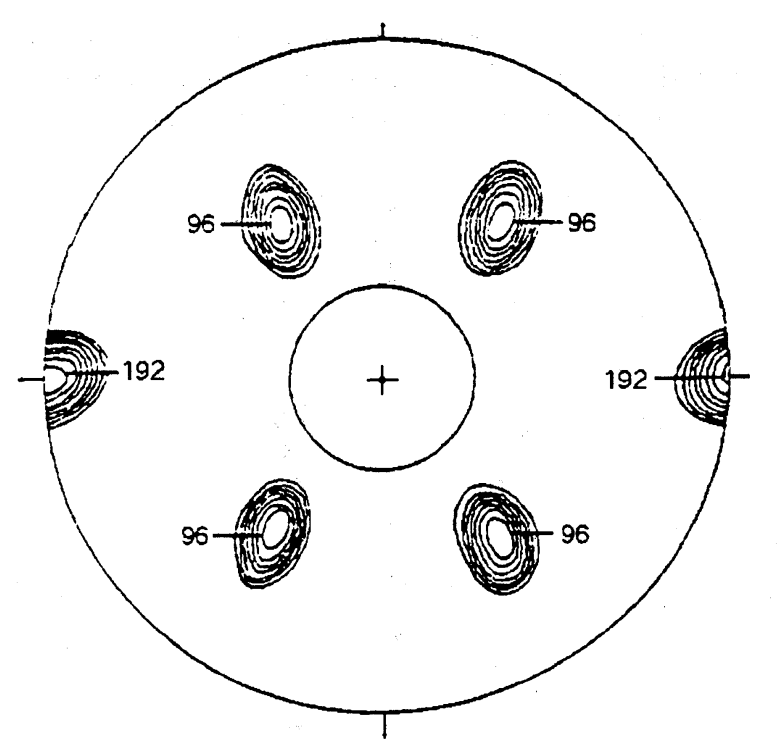

Fig. 10. Pole density stereogram of (110) pole of silicon steel specimen recrystallized for $1 \mathrm{~min}$ at $980^{\circ} \mathrm{C}$ [25].

normal to both [1 $1 \overline{1} 0]$ and [11 $\overline{3}]$ directions, is parallel to the [110] direction of recrystallized grains, the [110] direction is commonly shared by the deformed matrix and recrystallized grains, whereby the minimum shuffling condition can be achieved. In conclusion, the maximum stress direction, [11 $\overline{3}$ ], and the [332] direction become parallel to the [001] and [110] directions in recrystallized grain, respectively. In other words, the [001] direction must be on the (110) plane.

It follows from the above geometrical relations that

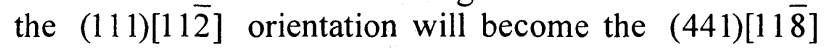
ori-entation after recrystallization. The symmetry yields another equivalent orientation, $(44 \overline{1})[\overline{1} 1 \overline{1} \overline{8}]$. The $(110)$ pole figure of the $\{441\}<118>$ orientation is shown in Fig. 8 along with the Goss orientation, $\{110\}<001>$. The $\{441\}<118>$ orientation is deviating from the Goss orientation by $10^{\circ}$. If each $\{441\}<118>$ orientation is represented by the Gauss type scattering with a half width angle of $12^{\circ}$, the calculated result is shown in Fig. 9. This is in very good agreement with the measured data in Fig.10, where the highest intensity poles are the same as those of the Goss orientation, even though it is not a real Goss orientation. It is also interesting to note that the rotation angle between (111)[112] and (441)[11 $\overline{8}$ ] about a common pole of [110] is calculated to be $25^{\circ}$ and the rotation angle between (111)[112] and (110)[001] about a common pole of [110] is $35^{\circ}$. This analysis was first made by Lee and Jeong [23]. This angular relation is often taken as an evidence for oriented growth theory for recrystallization $[26,27]$, in which the relation is attributed to the grain boundary mobility anisotropy. However, the angular relation is also obtained by the present method. Therefore, the angular relation may not be attributed to the grain boundary mobility anisotropy.

\section{SUMMARY}

The Goss orientation, $\{110\}<001>$, formed near the surface layer of silicon steel during rolling at elevated temperatures is thermally stable. The Goss orientation rotates toward the $\{111\}<112>$ orientation during plane strain cold rolling. The $\{111\}<112>$ orientation is calculated to change into the $\{441\}<118>$ after recrystallization. Representing each $\{441\}<118>$ by the Gauss type scattering gives rise to the Goss textures.

Acknowledgment - This work was supported by National Research Laboratory for Texture Control, Seoul National University.

\section{REFERENCES}

1. S. H. Lee and D. N. Lee, Mater. Sci. Eng., A249 (1998) 84.

2. C. G. Dunn, Acta metall., 2 (1954) 173.

3. D. Raabe and K. Lücke, Materials Science Forum, 157-162 (1994) 597.

4. Y. Inokuti et al., Proc. of 6th Int. Conf. On Textures Of Materials, (ed. by S.-I. Nagashima), The Iron and Steel Institute of Japan, Tokyo (1981) 948.

5. M. Matsuo, ISIJ International, 29 (1989) 809.

6. D. N. Lee, Scripta Metall. Mater., 32 (1995) 1689.

7. Y. B. Park, D. N. Lee and G. Gottstein, Mater. Sci. Tech., 13 (1997) 289

8. D. N. Lee, Texture and Microstructures, 26-27 (1996) 361.

9. S.-H. Hong, H.-T. Jeong, and D.N. Lee, Mater. Sci. Eng., A229 (1997) 174.

10. C.-H. Choi and D. N. Lee, Metall. Mater. Trans., 28A (1997) 2217

11. D. N. Lee and H.-T. Jeong, Mater. Sci. Eng., A269 (1999) 49.

12. D. N. Lee, S. Kang, and J. Yang, Plating and Surface Finishing, 82 (March 1995) 76.

13. D. N. Lee, Metals and Materials, 2 (1996) 121.

14. H. S. Nam and D. N. Lee, Proc. PRICM3, (ed. by M.A. Imam, R. DeNale, S. Hanada, Z. Zhong, and D. N. Lee), TMS, Hawaii (1998) 205.

15. A.P. Sutton and R.W. Ballufi, Interfaces in Crystalline Materials, Clarendon Press, Oxford (1996) 115

16. M. Zehetbaner, Acta metall. mater., 41 (1993) 589.

17. H.-J. Bunge, Texture Analysis in Materials Science, Butterworth, Guildford (1982).

18. J.W. Kwon, C.-H. Choi, and D. N. Lee, 5th Symp. Plasicity and Its Current Applications, (ed. by A.S. Khan), Elsevier, Amsterdam (1995) 157.

19. G.I. Taylor, J. Inst. Met., 62 (1938) 307.

20. J.F.W. Bishop and R. Hill, Phil. Mag., 42 (1951) 414.

21. M. Renouard and M. Wintenberger, C. R. Hebd. Seanc. Acad. Sci. Ser., B 292 (1981) 385

22. F.J.Humphreys and M.Hatherly, Recrystallization and Related Annealing Phenomena, Pergamon Press, New York (1995) 19.

23. D.N. Lee and H.-T. Jeong, Scripta Mater., 38 (1998) 1219

24. C.N. Reid, Deformation Geometry for Materials Scientists, Pergamon Press, Oxford (1973).

25. C.G. Dunn, Acta metall., 2 (1954) 173

26. G. Ibe and K. Lücke, Arch. Eisenhuyyenwes., 39 (1968) 693.

27. K. Lücke and M. Holscher, Texture and Microstructure., 1418 (1991) 585. 